\title{
Detecting intention to grasp during reaching movements from EEG
}

\author{
Luca Randazzo Iñaki Iturrate Ricardo Chavarriaga Robert Leeb José del R. Millán
}

\begin{abstract}
Brain-computer interfaces (BCI) have been shown to be a promising tool in rehabilitation and assistive scenarios. Within these contexts, brain signals can be decoded and used as commands for a robotic device, allowing to translate user's intentions into motor actions in order to support the user's impaired neuro-muscular system. Recently, it has been suggested that slow cortical potentials (SCPs), negative deflections in the electroencephalographic (EEG) signals peaking around one second before the initiation of movements, might be of interest because they offer an accurate time resolution for the provided feedback. Many state-of-the-art studies exploiting SCPs have focused on decoding intention of movements related to walking and arm reaching, but up to now few studies have focused on decoding the intention to grasp, which is of fundamental importance in upper-limb tasks. In this work, we present a technique that exploits EEG to decode grasping correlates during reaching movements. Results obtained with four subjects show the existence of SCPs prior to the execution of grasping movements and how they can be used to classify, with accuracy rates greater than $70 \%$ across all subjects, the intention to grasp. Using a sliding window approach, we have also demonstrated how this intention can be decoded on average around $400 \mathrm{~ms}$ before the grasp movements for two out of four subjects, and after the onset of grasp itself for the two other subjects.
\end{abstract}

\section{INTRODUCTION}

Brain-computer interfaces (BCI) can be used for restoring lost motor functionalities in people affected by neurological disorders or with limited motor abilities due to traumatic events or pathological conditions such as spinal cord injuries or cerebrovascular accidents. In BCI-based systems, correlates of user's motor intentions are decoded from the brain electrical activity and can be then used as control signals for motor assistive devices [1].

Recently, the usefulness of these systems as rehabilitation tools has also been demonstrated [2]. Here, decoded signals can trigger a robotic rehabilitation device, creating a closedloop control system that translates the user's intention into a motor action, ultimately aiming at triggering learning processes by promoting brain plasticity. In these scenarios, an aspect of utmost importance is that of decoding neural imprints associated to movement attempts in order to actuate the robotic device accordingly. A reliable decoding can in fact create a strong sense of ownership over the artificial system, strongly influencing the outcomes of the proposed therapy. Furthermore, the activation of the artificial device upon constrained timing has been shown to meaningfully

Authors are with the Chair in Brain-Machine Interface (CNBI), Center for Neuroprosthetics (CNP) and École Polytechnique Fédérale de Lausanne (EPFL). email: luca.randazzo@epfl.ch. This work has been partially supported by Swiss NCCR Robotics. I.I. also acknowledges support from the EPFL Fellows fellowship programme co-funded by Marie Curie, FP7 Grant agreement no. 291771. influence the degree of functional recovery [3].

Slow cortical potentials (SCPs), slow negative deflections in the electroencephalographic (EEG) signals appearing on the motor cortex around one second before the initiation of movements, have been recently proposed for use within these contexts. A great advantages of these preparatory signals is that they can be detected before the actual movement, a key aspect when providing time-contingent feedback [4]. SCPs have been shown to appear both in healthy and motor injured people in a variety of tasks as upper-limb reaching movements [5], [6] and in lower-limbs motion initiation [7], among others.

So far, however, these studies have mainly focused on initiation of movements from a rest condition, while the neural correlates related to evolving movements are yet to be studied. One such movement is that of grasping, which is usually performed after a reaching movement. In rehabilitation and assistive scenarios, a reliable decoding of the intention to grasp while performing a reaching movement might allow to create more natural upper-limb motor therapies and assistive devices, enabling typical activities of daily living (ADL) as reach-to-grasp tasks. Several works have shown how cortical activity can be used to decode correlates of grasping tasks, with an emphasis on the classification of different grasp types using electrocorticography (ECoG) [8] or EEG [9]. Similarly, one work has shown the possibility of decoding the onset of grasp using ECoG [10].

In this work, we present a method for decoding the intention to grasp during reaching movements using EEG. To this end, and following previous studies on ECoG [8], [10], an experimental upper-limb task was designed to induce selfpaced reach-to-grasp movements and the EEG data recorded during experiments on four subjects were analyzed in order to assess the feasibility of the proposed decoding algorithm. The results showed the existence of slow cortical potentials prior to the execution of grasping movements and the feasibility of their single-trial classification.

\section{Methods}

\section{A. Experimental Protocol}

Following previous works on detection of grasping correlates using ECoG [8], [10], we designed an experimental protocol to induce self-paced reach-to-grasp movements, executed without any external cue in order to better simulate hand motor tasks during ADL.

During the experiment, participants were comfortably seated on a chair facing an object that they had to grasp. The protocol is shown in Figure 1. For each trial, subjects rested their dominant (right) hand on a button on the table. 
Whenever they wanted (but waiting at least 2 seconds after the previous trial), they performed a reaching movement towards an object placed at around $50 \mathrm{~cm}$ of distance. The object (an ordinary gym weight) could be grasped in two different positions (corresponding to two different arm-hand configurations) and using two different types of grasps (power grasp and precision pinch). The type of grasp was freely chosen by the subject at each trial. After grasping the object, subjects were instructed to lift it, place it back to its original position and go back with their hand to the rest position. Every 100 trials, the object was repositioned to a different place in order to remove any laterality confounds. Subjects were asked to restrain eye movements or blinks during the reaching and grasping states.

Four right-handed male healthy subjects (S1-S4, mean aged $30 \pm 4$ years), with no known history of neurological abnormalities or musculoskeletal disorders, participated in the experiment. Approximately 400 trials per subject were recorded. Trials where the rest phase lasted less than 2 seconds (around $5 \%$ of the total number) were removed from the recording. For each subject, recordings were performed in a single session that lasted approximately 2 hours including EEG setup and removal. Movement initiation and grasping onset were synchronized with the EEG by means of hardware triggers generated when the user grasped the object (see Figure 1b).

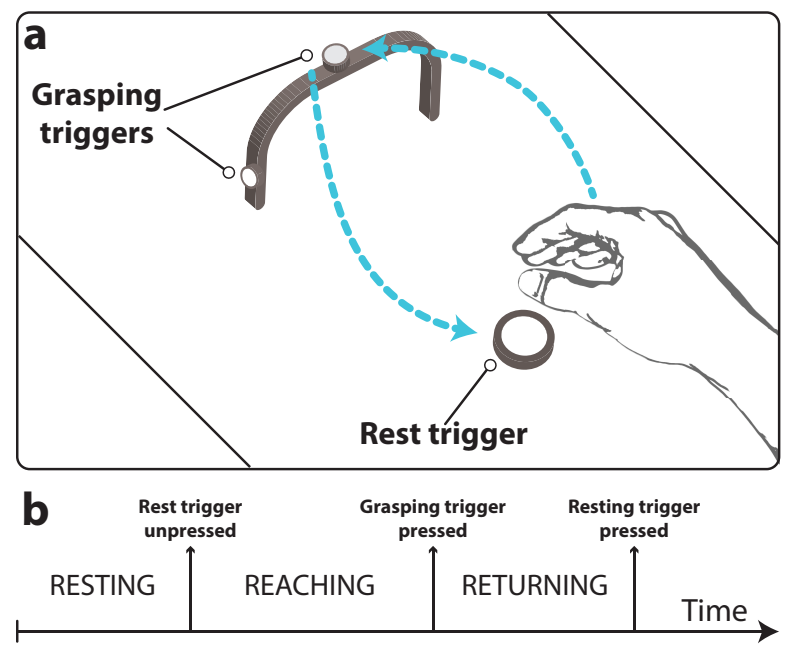

Fig. 1. Experimental protocol. (a) Schematic illustration of the setup. For each trial, subjects started with their dominant hand on the rest trigger, where they had to stay at least for 2 seconds. Then, they reached and grasped the object (using a power grasp or precision pinch, in two different positions signaled by two triggers), lifted the object and returned with their hand to the rest trigger. (b) Timeline of a single trial. $0 \mathrm{~ms}$ indicates the onset of grasp.

\section{B. Data Recording}

During the experiments, EEG activity was recorded using a BioSemi system with 64 active electrodes, equally distributed over the scalp following the 10/20 international system. EEG data were recorded at a sampling rate of $2048 \mathrm{~Hz}$, and downsampled to $256 \mathrm{~Hz}$. Additionally, 3 monopolar electrooculography (EOG) channels were recorded (placed above the nasion, and on the outer canthi of the left and right eyes). These channels were used to compute horizontal and vertical EOG, in order to remove the EOG components from the EEG using a regression algorithm [11]. Both the rest position and the object used during the experiment were sensorized in order to generate hardware triggers corresponding to the different events, allowing to record data having a precise time synchronization between EEG signals and movement onsets.

\section{Offline classification}

An offline classification was first tested on the recorded data. To this end, EEG data were filtered in the range $[0.1,1] \mathrm{Hz}$ (non-causal $2^{\text {nd }}$ order Butterworth filter) and common-average re-referenced (CAR).

Data were then epoched based on the onset of grasp, defined as the moment when the subjects pressed either grasping trigger (see Figure 1a). Epochs containing artifacts (where the amplitude of one of the channels exceeded $100 \mu \mathrm{V})$ were excluded from the analysis. From these epochs, two classes were created: rest class, with samples taken from the time window $[-3000,-2250] \mathrm{ms}$, and intention to grasp class, from the time window $[-750,0] \mathrm{ms}$ (where $0 \mathrm{~ms}$ represents the onset of grasp).

For each of the two classes, time features were extracted from 9 channels over the contra-lateral motor cortex (FC3, $\mathrm{FC} 1, \mathrm{FCz}, \mathrm{C} 3, \mathrm{C} 1, \mathrm{Cz}, \mathrm{CP} 3, \mathrm{CP} 1$ and $\mathrm{CPz}$ ), with features consisting of the electrical amplitude recorded by the EEG electrodes.

Principal component analysis was then applied to retain $95 \%$ of the variance on the features matrix. Classification was performed using a linear discriminant classifier (LDA) and the decoding accuracy evaluated with a 10-fold crossvalidation procedure on the whole dataset.

\section{Pseudo-online classification: Sliding window}

In order to assess the performance that could be obtained with an online decoding algorithm, we performed a sliding window classification analysis. In this case, to better simulate the conditions of an online experiment, EEG data were filtered with a casual filter in the range $[0.1,1] \mathrm{Hz}$ (causal $2^{\text {nd }}$ order Butterworth filter).

After filtering, data were separated in training and testing sets, $75 \%$ and $25 \%$ of the data respectively and keeping the temporal structure of the trial. The classifier was learned from the training dataset using the same parameters used for the offline analysis. Then, we applied a sliding window method (with steps of $62.5 \mathrm{~ms}$ ) to classify the testing set. The time range considered for evaluating the sliding window approach was from $5000 \mathrm{~ms}$ before the onset of grasp and up to $1000 \mathrm{~ms}$ after it.

\section{RESULTS}

\section{A. Neurophysiological results}

Figure 2 shows the grand averages across trials for three channels $(\mathrm{C} 3, \mathrm{C} 1$ and $\mathrm{Cz})$ over the left cortex, contralateral to the motor task. For the sake of simplicity, only the averages obtained for subject S1 are shown. As can be 


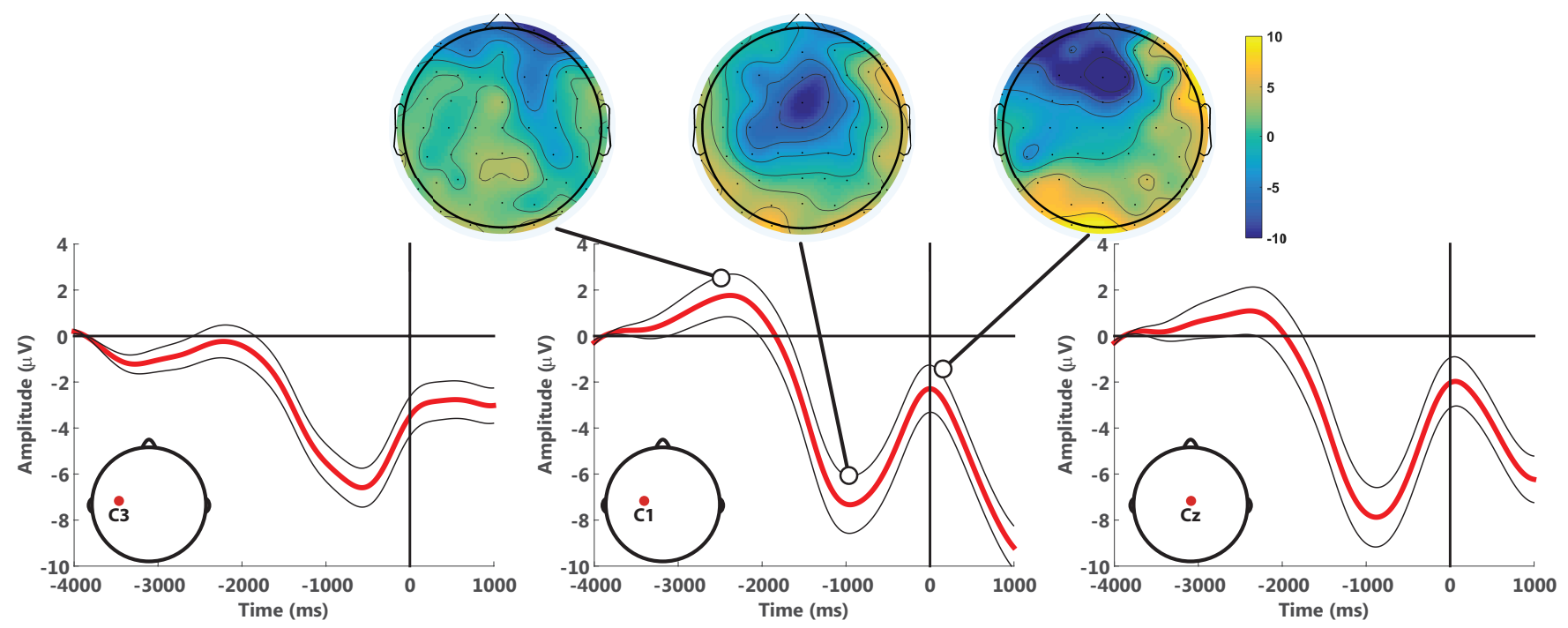

Fig. 2. Averaged signals (in bold red) across all trials for subject S1, for channels C3, C1, Cz. Thinner black lines represent the standard error of the mean. Time $0 \mathrm{~ms}$ corresponds to the onset of grasp.

seen in topographic interpolations, a negativity is mainly developing over the contra-lateral and central motor cortex around $1 \mathrm{~s}$ before the onset of grasp. Importantly, these results are consistent with other studies related to movement anticipation in EEG [6], and with detection of onset of grasp from ECoG [10], suggesting the existence of neural correlates of intention to grasp under a constrained task.

\section{B. Offline classification}

Figure 3 shows the results of the offline classification algorithm, reported in terms of accuracy rates for both classes, for each subject. High accuracy rates (above 70\%) were obtained for all the subjects, with average rates of $73.72 \% \pm 1.85$ and $78.22 \% \pm 2.29$ for the rest and intention to grasp classes respectively.

\section{Sliding window}

Figure 4 shows the results of the classification performed on the testing dataset using the sliding window method. For each subject, the mean of the posterior probability for the intention to grasp class across all the trials is reported. As visible here, the classifier was able to achieve reliable abovechance $^{1}$ classification accuracies $(>65 \%)$ up to $1 \mathrm{~s}$ before the onset of grasping for subjects S1 and S2. Despite subjects S3 and $\mathrm{S} 4$ presented lower results, in their cases discriminable information was also present after the actual onset of grasp.

To further interpret these results, Table I reports, for each subject, the peak probabilities and their latency (with respect to the onset of grasp), computed from the results shown in Figure 4. For subjects S1 and S2, the peak accuracy rates were achieved more than $300 \mathrm{~ms}$ before the onset of grasping, reaching a 0.67 and 0.78 of probability respectively. For subject S3, although the classification accuracy peaked after

\footnotetext{
${ }^{1}$ Chance level was computed empirically by shuffling the labels 1000 runs and then averaging across runs.
}

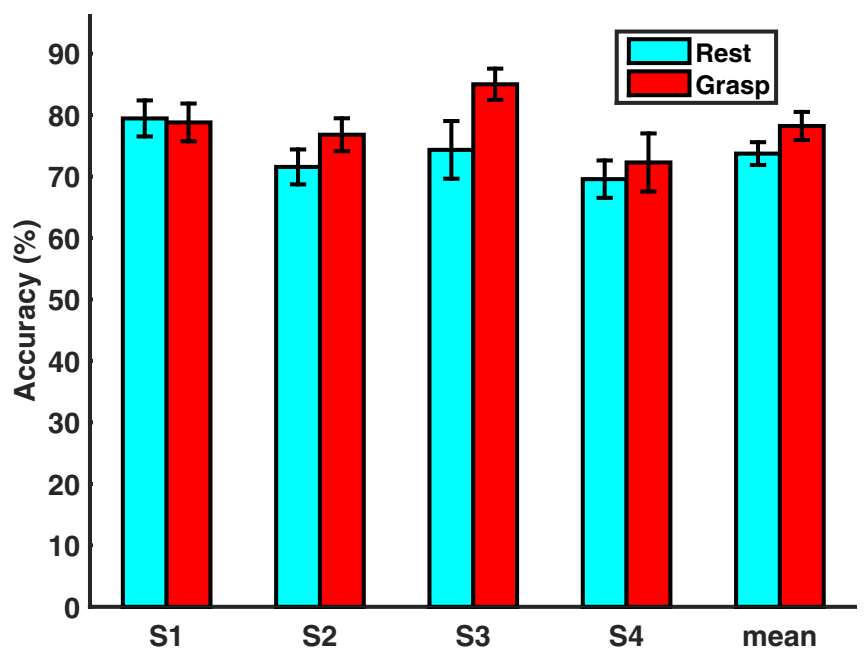

Fig. 3. Offline classification results. For each subject, the mean and standard error of the mean of the classification accuracies (evaluated within a 10 fold cross-validation procedure) are reported for both classes, namely rest (light blue) and intention to grasp (red). Additionally, the mean accuracy rates across all subjects for both classes are reported on the right-most side. The horizontal dashed line represents the chance level, computed empirically by shuffling the labels of the classes during the classification.

the onset itself, above chance classification could be achieved around $100 \mathrm{~ms}$ before the onset. For subjects S3 and S4, the classifier was able to decode the intention to grasp peaking after the onset of grasp, at 438 and $125 \mathrm{~ms}$ respectively.

\section{CONCLUSIONS AND FUTURE WORK}

This preliminary study suggests the existence of neural correlates of intention to grasp, in able-bodied subjects. We have shown the possibility of decoding these grasping correlates while performing a reaching movement with offline mean accuracies higher than $70 \%$ across all the subjects. Furthermore, emulating an online decoding by using a sliding 

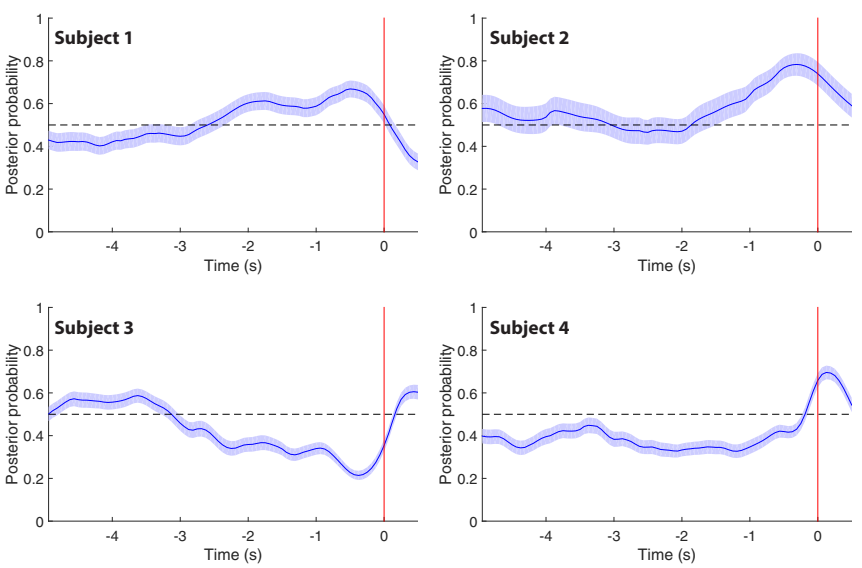

Fig. 4. Sliding window classification results. For each subject, it is shown the mean across all testing trials (blue line) and standard error of the mean (shaded area) of the posterior probability of the intention to grasp class. The red line corresponds to the onset of grasp, the dashed horizontal line represents the chance level.

TABLE I

SLIDING WINDOW CLASSIFICATION RESULTS

\begin{tabular}{rccccc}
\hline & S1 & S2 & S3 & S4 & mean \pm std \\
\hline Peak probability & 0.67 & 0.78 & 0.61 & 0.70 & $0.69 \pm 0.07$ \\
Delay $(\mathbf{m s})$ & -500 & -313 & 438 & 125 & $-62.50 \pm 424$ \\
\hline
\end{tabular}

window approach, we were able to predict the intention to grasp around $400 \mathrm{~ms}$ before the grasp movement for two out of four subjects, with the other two subjects showing discriminant information after the onset itself.

A main limitation of this study could be the presence of motor confounds during the reaching period, different from those of grasping. Notice, however, that this may be an unavoidable condition under real conditions and natural tasks such as reach-to-grasp movements. Additionally, the recordings were made under a well-controlled laboratory condition, and further work remains to be done to test whether these correlates are present under more natural, lesscontrolled scenarios such as during activities of daily living or rehabilitation therapies. Results also need to be evaluated on potential BCI end users, mainly stroke and spinal cord injured people in order to evaluate their actual usefulness. Nonetheless, past results have already shown the existence of similar slow potentials on stroke patients [6], and their possible applicability for rehabilitation strategies [4].

Overall, although preliminary, these results are very promising as they hold the promise of enabling a more natural and intuitive control of artificial devices as prostheses and orthoses for motor impaired people, both in rehabilitation and assistive scenarios. Ultimately, we aim at improving functional recovery in motor rehabilitation therapies by achieving seamless user-machine interaction.

\section{REFERENCES}

[1] J.d.R. Millán et al., "Combining brain-computer interfaces and assistive technologies: state-of-the-art and challenges," Frontiers in neuroscience, vol. 4, 2010.

[2] S.R. Soekadar, N. Birbaumer, M.W. Slutzky, and L.G. Cohen, "BrainMachine Interfaces In Neurorehabilitation of Stroke.," Neurobiology of disease, 2014

[3] A. Ramos-Murguialday et al., "Brain-machine interface in chronic stroke rehabilitation: a controlled study," Annals of neurology, vol 74, no. 1, pp. 100-108, 2013.

[4] N. Jiang, N. Mrachacz-Kersting, R. Xu, K. Dremstrup, and D. Farina, "An accurate, versatile, and robust brain switch for neurorehabilitation," in Brain-Computer Interface Research, pp. 47-61. Springer, 2014.

[5] E. López-Larraz, L. Montesano, A. Gil-Agudo, and J. Minguez, "Continuous decoding of movement intention of upper limb self-initiated analytic movements from pre-movement eeg correlates," Journal of neuroengineering and rehabilitation, vol. 11, no. 1, pp. 153, 2014.

[6] E. Lew, R. Chavarriaga, S. Silvoni, and J.d.R Millán, "Detection of self-paced reaching movement intention from eeg signals," Frontiers in Neuroengineering, vol. 5, no. 13, 2012.

[7] I.K. Niazi, N. Jiang, O. Tiberghien, J.F. Nielsen, K. Dremstrup, and D. Farina, "Detection of movement intention from single-trial movement-related cortical potentials," Journal of neural engineering, vol. 8, no. 6, 2011.

[8] T. Pistohl, A. Schulze-Bonhage, A. Aertsen, C. Mehring, and T. Ball, "Decoding natural grasp types from human ECoG," Neuroimage, vol. 59, no. 1, pp. 248-260, 2012.

[9] H. Agashe, A.Y. Paek, Y. Zhang, and J.L Contreras-Vidal, "Global cortical activity predicts shape of hand during grasping," Frontiers in Neuroscience, vol. 9, no. April, pp. 1-11, 2015.

[10] T. Pistohl, T.S.B. Schmidt, T. Ball, A. Schulze-Bonhage, A. Aertsen, and C. Mehring, "Grasp detection from human ECoG during natural reach-to-grasp movements," PloS one, vol. 8, no. 1, pp. e54658, 2013.

[11] A. Schlögl, C. Keinrath, D. Zimmermann, R. Scherer, R. Leeb, and G. Pfurtscheller, "A fully automated correction method of EOG artifacts in EEG recordings," Clinical neurophysiology, vol. 118, no. 1, pp. 98-104, 2007. 